\title{
Development of Benzodithiophene-Based A-D-A Small Molecules with Different Acceptor End Groups for Efficient Organic Solar Cells
}

\author{
JIA Guoxiao ${ }^{1}$, ZHANG Shaoqing $1{ }^{1,}$, , YANG Liyan ${ }^{2}$, HE Chang ${ }^{2}$, FAN Huili $1{ }^{1,}$, , HOU Jianhui $1,2,{ }^{*}$ \\ ${ }^{1}$ School of Chemical and Biological Engineering, University of Science and Technology Beijing, Beijing 100083, P. R. China. \\ ${ }^{2}$ State Key Laboratory of Polymer Physics and Chemistry, Beijing National Laboratory for Molecular Sciences, Institute of \\ Chemistry, Chinese Academy of Sciences, Beijing 100190, P. R. China
}

\begin{abstract}
In recent years, organic solar cells (OSCs) have attracted increasing attention, and the power conversion efficiency (PCE) of OSCs has markedly improved. To enhance the photovoltaic properties of OSCs, it is important to develop the donor materials in the light-harvesting layer, which mainly include conjugated polymers and small molecules (SMs). Compared with polymeric materials, small-molecule materials have been widely investigated for their superior characteristics, such as well-defined molecular structures that can provide good batch-to-batch reproducibility. In this work, we synthesized three SM donor materials with theacceptor-donor-acceptor (A-D-A) structure by employing the trialkylthienyl-substituted

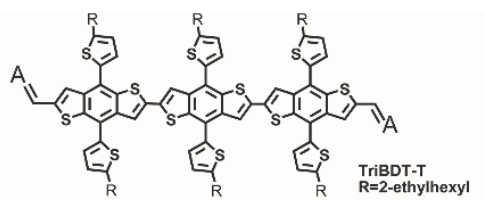
benzodithiophene (TriBDT-T) unit as the D-core unit, and rhodanine (RN), cyano-rhodanine (RCN), and 1,3-indanone (IDO) as the A end groups, respectively. The optical properties, molecular energy levels, and thermogravimetic characteristics of the three SMs were studied; moreover, the blend morphologies and photovoltaic properties of the devices by employing the non-fullerene (NF) acceptor, IT-4F, were systematically investigated. The results showed that 1) the three SMs exhibit good thermal stabilities as evinced by thermogravimetric analysis (TGA), and all decomposition temperatures exceeded $410^{\circ} \mathrm{C}$; 2) They all exhibit strong and broad absorption in the visible light range (300-700 nm), and show similar molar extinction coefficients; 3 ) the HOMO levels are $-5.47 \mathrm{eV},-5.54 \mathrm{eV}$, and $-5.44 \mathrm{eV}$ for RN, RCN, and IDO, respectively, implying the clear influence of the different end groups for the energy levels of the A-D-A-type SMs; the slight differences in the optical and electrochemical properties of the corresponding donor material could be attributed to the different electron-withdrawing ability of the A-type end groups. When studying the photovoltaic properties, interestingly, the RN:IT$4 \mathrm{~F}$ blend was found to form fibrillar-like aggregates with appropriate size, and the corresponding devices exhibited desirable short circuit current $\left(J_{\mathrm{sc}}\right)$ and thus the highest PCE value of $9.25 \%$; however, large-size aggregates were formed in the RCN:IT-4F and IDO:IT-4F blend films, resulting in a much lower $J_{\text {sc }}$ and fill factor (FF), and the PCE of the corresponding devices were only around $6 \%$. In summary, by introducing RN, RCN, and IDO as the A units, we synthesized a class of TriBDT-T based A-D-A type SMs. This study shows that terminal A units exert influence on the absorption spectra, molecular energy level, and morphologies after blending with the acceptor material, and hence, the corresponding devices exhibit significant differences in photovoltaic performance. This work also provides useful information for the molecular design of SM donor materials.
\end{abstract}

Key Words: Small molecule donor; Terminal modification; Organic solar cells; Non-fullerene acceptor; AcceptorDonor-Acceptor structure

\footnotetext{
Received: November 8, 2017; Revised: November 30, 2017; Accepted: December 1, 2017; Published online: December 6, 2017.

${ }^{*}$ Corresponding authors. Emails: shaoqingz@iccas.ac.cn (Z.S.); fanhl@sas.ustb.edu.cn (F.H.); hjhzlz@iccas.ac.cn (H.J.).

The project was supported by National Natural Science Foundation of China (2170040201), National Science and Technology Major Project of the Ministry of Science and Technology of China (2016YFC0700603), and the Fundamental Research Funds for the Central Universities, China (FRF-TP-17-009A1). 国家自然科学基金(2170040201)，国家科技重大专项(2016YFC0700603)和中央高校基本科研业务费专项资金(FRF-TP-17-009A1)资助项目 (C) Editorial office of Acta Physico-Chimica Sinica
} 


\title{
A-D-A 型小分子电子给体光伏材料的端基修饰及其光伏性能
}

\author{
贾国骁 ${ }^{1}$, 张少青 $1^{,}$, 杨丽燕 ${ }^{2}$, 何畅 ${ }^{2}$, 范慧俐 $1,{ }^{*}$, 侯剑辉 $1,2,{ }^{*}$ \\ 1 北京科技大学化学与生物工程学院, 北京 100083 \\ 2 高分子物理与化学国家重点实验室, 北京分子科学国家实验室, 中国科学院化学研究所, 北京 100190
}

\begin{abstract}
摘要: 有机太阳能电池(OSCs)活性层中的给体材料主要包括共轭聚合物与有机小分子, 由于有机小分子给体具有结构确 定、易于提纯、重复性高等独特的优势, 近年来受到研究工作者的广泛关注。本工作中, 我们采取具有良好共平面性的 三联苯并二噻吩(TriBDT- $T$ )为推电子(D)中心共轭单元, 分别以罗丹宁 $(R N)$ 、萻基罗丹宁 $(R C N)$ 和1,3-狮二酮(IDO)为拉电 子(A)共轭端基, 设计并合成了三种具有A-D-A型结构的小分子给体材料TriBDT-T-RN、TriBDT-T-RCN和TriBDT-T-IDO。 我们对比研究了三种端基对其热分解温度、吸收光谱和分子能级等基本性能的影响, 并分别将三种小分子给体与非富勒 烯型受体材料IT-4F共混制备器件, 详细研究了活性层形貌与光伏性能之间的关系。结果表明, 不同的A型端基对小分子 给体材料的光学性能、电化学性能、光伏器件中活性层的微观形貌以及能量转换效率(PCE)产生显著影响。基于TriBDT-
\end{abstract} T-RN:IT-4F、TriBDT-T-RCN:IT-4F和TriBDT-T-IDO:IT-4F的光伏器件的能量转换效率分别为 $9.25 \% 、 6.31 \%$ 和 $6.18 \%$ 。

关键词：小分子给体；端基修饰；有机太阳能电池；非富勒烯受体；A-D-A结构 中图分类号: 0646

\section{1 引言}

相较于传统的无机太阳能电池, 有机太阳能 电池(OSCs)具备成本低、重量轻、可通过溶液加工 方式制备柔性器件等诸多优点, 已经成为具有重 要应用前景的太阳能利用方式之一 ${ }^{1-3}$ 。能量转换 效率是衡量有机太阳能电池光伏性能的重要标 准, 自 1995 年俞刚等 ${ }^{4}$ 发明了具有本体异质结结 构(BHJ)的 OSC 以来, 用于此类电池的光伏材料 和器件制备工艺得到持续的发展, 采用共轭聚合 物电子给体和小分子电子受体材料构建 BHJ 光伏 活性层的电池能量转换效率(PCE) 已经达到 $13 \%$ 以上 ${ }^{5}$ 。

活性层材料是决定有机太阳能电池能量转换 效率的重要因素之一 6,7 。相比于共轭聚合物给体 材料, 有机小分子具有化学结构十分确定、纯度评 价标准明确、批次差别性小的优点, 因此在对分子 结构与光伏性能之间关系的研究工作中, 针对小 分子给体材料的结构设计工作至关重要。目前, 具 有拉-推-拉(A-D-A)电子作用的共轭结构已经被广 泛应用到小分子给体与受体材料的分子设计中, 并 取得了大量具备优异光伏性能的活性层材料 ${ }^{8-15}$ 。 在这种结构中, 人们可以通过对推电子(D)单元 ${ }^{16-20}$ 、 拉电子(A)单元 ${ }^{21-25}$ 和二者之间的联结方式的调 节 ${ }^{26-29}$ 来实现对材料吸收光谱和分子能级进行有效 调控。与 A-D-A 型受体材料相比 ${ }^{30-34}$, A-D-A 型 给体的吸收光谱相对蓝移, 是由于它们的给体单 元和末端基团之间推拉电子作用不同所致。因此, A-D-A 型给体和受体的组合可以提供宽吸收光谱
和适当的分子能级, 这是实现高效的光吸收和高 输出电压的两个关键因素。因此, 采用 A-D-A 型 小分子给体与受体共混制备太阳能电池, 是一项 具有意义的工作。

在本工作中, 我们以具有良好共平面性的三 联苯并二噻吩(TriBDT)结构为 D 单元 ${ }^{19,35,36}$, 分别 以罗丹宁 $(\mathrm{RN})$ 、氧基罗丹宁 $(\mathrm{RCN})$ 和 1,3-狮二酮 (IDO)为 $\mathrm{A}$ 单元, 设计并合成了具有 A-D-A 结构 的带有上述三种不同端基的小分子给体材料, 细 致研究了三种端基对其热分解温度、吸收光谱、分 子能级等性能的影响。随后我们将三种小分子给 体与非富勒烯型受体材料 IT-4F 共混制备器件, 详 细研究了三种端基对于小分子材料与受体材料共 混制备的活性层形貌与光伏性能之间的关系。

\section{2 实验部分}

\section{1 材料合成}

化合物 1-4 均购自朔纶有机光电科技(北京) 有限公司, 实验中用到的其他溶剂与化合物均购 自百灵威科技有限公司。三个带有不同 $\mathrm{A}$ 型端基 的小分子给体材料 TriBDT-T-RN、TriBDT-T-RCN 和 TriBDT-T-IDO(下文中分别简称为 RN $\mathrm{RCN}$ 和 IDO)的合成步骤如图 1 所示与结构表征结果如 下。

$\mathbf{R N}$ : 将化合物 $1(180 \mathrm{mg}, 0.1 \mathrm{mmol})$ 与 $2(0.16$ $\mathrm{g}, 1 \mathrm{mmol}$ )称量至 $100 \mathrm{~mL}$ 双口瓶中, 用氩气将体 系保护起来。将 $20 \mathrm{~mL}$ 无水氯仿用注射器注入反 应瓶中, 反应物溶解后再向其中注入 $0.3 \mathrm{~mL}$ 哌啶, 


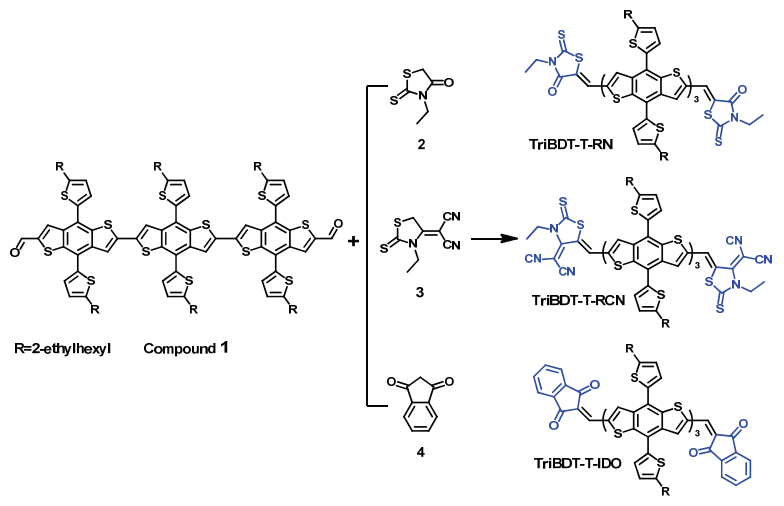

图 1 本工作中三种小分子给体的合成路线与分子结构

Fig. 1 The synthetic route and the molecular structure of the three small molecules (SMs).

随后将反应升温至 $65{ }^{\circ} \mathrm{C}$ 搅拌过夜。反应完成后 将体系降温, 向其中加入 $50 \mathrm{~mL}$ 去离子水, 并用 $50 \mathrm{~mL}$ 氯仿萃取两次, 有机相合并浓缩后用柱层 析的方式以纯二氯甲烷为淋洗剂进行提纯, 最终 得到的产物为紫红色固体, 产率约为 $50 \%$ 。

${ }^{1} \mathrm{H} \mathrm{NMR}\left(\mathrm{CDCl}_{3}, 300 \mathrm{MHz}\right), \delta: 7.74(\mathrm{~s}, 2 \mathrm{H})$, $7.55(\mathrm{~s}, 2 \mathrm{H}), 7.50(\mathrm{~s}, 2 \mathrm{H}), 7.42(\mathrm{~s}, 2 \mathrm{H}), 7.29$ $7.26(\mathrm{~m}, 6 \mathrm{H}), 6.94-6.90(\mathrm{~m}, 6 \mathrm{H}), 4.07-4.05(\mathrm{~m}$, $4 \mathrm{H}), 2.96-2.91(\mathrm{~m}, 12 \mathrm{H}), 1.80-1.77(\mathrm{~m}, 6 \mathrm{H}), 1.54-$ $1.46(\mathrm{~m}, 48 \mathrm{H}), 1.06-1.02(\mathrm{t}, 6 \mathrm{H}), 0.98-0.93(\mathrm{~m}$, $36 \mathrm{H})$ 。

元素分析结果: $\mathrm{C}_{114} \mathrm{H}_{132} \mathrm{~N}_{2} \mathrm{O}_{2} \mathrm{~S}_{16}$ 的理论值 (\%): C 65.98; H 6.41; N 1.35。实测值(\%): C 65.84; H 6.40; N 1.35。

$\mathbf{R C N}$ ：反应步骤与 $\mathrm{RN}$ 基本相同，当使用柱 层析方式对产物进行提纯时, 需要使用二氯甲烷 与四氢呋喃体积比为 $10: 1$ 的混合溶剂作为淋洗 剂。产物为紫黑色固体, 产率约为 $40 \%$ 。

${ }^{1} \mathrm{H} \mathrm{NMR}\left(\mathrm{CDCl}_{3}, 400 \mathrm{MHz}\right), \delta: 7.82(\mathrm{~s}, 2 \mathrm{H})$, $7.80(\mathrm{~s}, 2 \mathrm{H}), 7.51(\mathrm{~s}, 2 \mathrm{H}), 7.47(\mathrm{~s}, 2 \mathrm{H}) 7.27(\mathrm{~m}$, $6 \mathrm{H}), 7.00-6.93(\mathrm{~m}, 6 \mathrm{H}), 4.20-4.18(\mathrm{~m}, 4 \mathrm{H}), 2.93$ $(\mathrm{m}, 12 \mathrm{H}), 1.78-1.76(\mathrm{~m}, 6 \mathrm{~h}), 1.51-1.33(\mathrm{~m}, 48 \mathrm{H})$, $1.03-0.94(\mathrm{~m}, 42 \mathrm{H})$ 。

元素分析结果: $\mathrm{C}_{120} \mathrm{H}_{132} \mathrm{~N}_{6} \mathrm{~S}_{16}$ 的理论值 (\%): C 66.38; H 6.13; N 3.87。实测值(\%): C 66.85; H 6.23; N 3.91。

IDO: 化合物 $1(287 \mathrm{mg}, 0.16 \mathrm{mmol})$ 与 $4(234$ $\mathrm{mg}, 1.6 \mathrm{mmol}$ )混合置于双口瓶中, 反应体系需用 氩气保护。加入溶剂无水氯仿 $30 \mathrm{~mL}$, 随后加入吡 啶 $0.5 \mathrm{~mL}$, 将反应升温至 $65{ }^{\circ} \mathrm{C}$ 过夜。反应后处 理方式与 $\mathrm{RN}$ 相同, 产物由柱层析方式提纯, 为暗 紫色色固体，产率约为 $40 \%$ 。

MOLDI-TOF $\left(\mathrm{C}_{122} \mathrm{H}_{130} \mathrm{O}_{4} \mathrm{~S}_{12}\right): m / z=2045.5$
元素分析结果: $\mathrm{C}_{122} \mathrm{H}_{130} \mathrm{O}_{4} \mathrm{~S}_{12}$ 的理论值 (\%): C 71.65; H 6.41。实测值(\%): C 71.49; H 6.45 。

\section{2 器件制备}

本工作中所使用的器件结构为: ITO/ $\mathrm{MoO}_{3}$ $(10 \mathrm{~nm})$ /活性层/A1 $(100 \mathrm{~nm})$ 。将 ITO 玻璃基板清 洗干净, 干燥后使用紫外-臭氧处理 $20 \mathrm{~min}$, 随后 移至充满氮气的手套箱内蒸镀 $\mathrm{MoO}_{3}$, 接着, 将小 分子给受体充分溶解在氯仿中, 旋涂到 $\mathrm{MoO}_{3}$ 层 上, 使用溶剂退火方式(Solvent Vapor Annealing, SVA) 对活性层形貌进行调控, 最后真空蒸镀铝电 极(约 $100 \mathrm{~nm}$ )。活性层溶液的制备条件详见结果 与讨论部分。

\section{3 表征测试方法}

热重分析(TGA)在 Pyris 1 TGA (PerkinElmer) 上进行测量。差示扫描量热(DSC)测试使用的是 DSC-Q200 (TA Instruments Inc.)。X-ray diffraction (XRD)使用的 Rigaku MiniFlex 600 测量。紫外可 见-可见吸收光谱(UV-Vis) 使用 Hitachi U-3100 UV-Vis 分光光度计进行测量。循环伏安测试(CV) 使用 CHI650D 电化学工作站: 使用 Pt 盘(工作电 极), $\mathrm{Pt}$ 丝(对电极)和 $\mathrm{Ag} / \mathrm{Ag}^{+}$电极(参比电极); 二 茂铁/二茂铁氧化还原对 $\left(\mathrm{Fc} / \mathrm{Fc}^{+}\right)$用作内标。外量子 效率(EQE)使用 QE-R3011 (Enli Technology Co., Ltd.) 测试。原子力显微镜 (AFM) 图像通过 Nanoscope III A (Vecoo) 以 Tapping 模式测量。透 射电子显微镜(TEM) 使用 JEM-2200FS 仪器在 2 $\mathrm{kV}$ 的加速电压下测得。

\section{3 结果与讨论}

\section{1 光学性质与分子能级}

三种小分子给体材料的光学性质通过 UV-Vis 吸收光谱进行了表征。表 1 中提供了三种小分子 的部分光谱数据; 图 2 为 RN、RCN、IDO 和受体 材料 IT-4F 分别溶于氯仿溶液中测得的溶液吸收 光谱(a)和固态薄膜的吸收光谱(b)。如图 2a 所示, 在氯仿溶液中, 三种有机小分子在可见光区 $(300-$ $700 \mathrm{~nm}$ )均具有强而宽的吸收, RN、RCN 和 IDO 溶液的最大吸收峰 $\left(\lambda_{\max }\right)$ 分别位于约 546、562 和 $576 \mathrm{~nm}$ 处, 说明在三种 A-D-A 型小分子给体材料 中, 给体单元 BDT 和受体单元 $(\mathrm{RN} 、 \mathrm{RCN} 、 \mathrm{IDO})$ 之间的分子内电荷转移(ICT) 效应有所不同, 即: 端基的拉电子作用越强，分子内的 ICT 效应越明 显, 因此吸收光谱相对红移 $24,34,37,38$ 。根据图 $2 b$ 中 薄膜的吸收边可以计算出 RN、RCN 和 IDO (629、 659 和 $694 \mathrm{~nm})$ 的光学带隙 $\left(E_{\mathrm{g}}^{\mathrm{opt}}\right)$ 分别为 $1.97 、 1.88$ 和 $1.78 \mathrm{eV}$, 表明不同的端基对小分子的光学带隙 
表 1 TriBDT-T 的光学性质和分子能级

Table 1 Optical and electrochemical properties of TriBDT-T.

\begin{tabular}{ccccccc}
\hline Materials & $\lambda_{\max } / \mathrm{nm}^{\mathrm{a}}$ & $\varepsilon /\left(\mathrm{L} \cdot \mathrm{mol}^{-1} \cdot \mathrm{cm}^{-1}\right)^{\mathrm{b}}$ & $\lambda_{\max } / \mathrm{nm}^{\mathrm{c}}$ & $\lambda_{\text {edge }} / \mathrm{nm}$ & $E_{\mathrm{g}}^{\text {opt}} / \mathrm{eV}$ & \\
\hline RN & 546 & $4.96 \times 10^{4}$ & 536 & 629 & 1.97 \\
RCN & 562 & $4.56 \times 10^{4}$ & 550 & 659 & 1.88 \\
IDO & 576 & $5.22 \times 10^{4}$ & 564 & 694 & -5.54 \\
\hline
\end{tabular}

${ }^{\mathrm{a}}$ Measured in dilute chloroform solution. ${ }^{\mathrm{b}}$ Maximum absorption coefficients in solution. ${ }^{\mathrm{c}}$ Measured on a quartz plate by donors cast from chloroform solution. ${ }^{\mathrm{d}}$ Estimated from the onset wavelength of the absorption spectra: $E_{\mathrm{g}}^{\text {optd }}=1240 / \lambda_{\text {edge }}$.
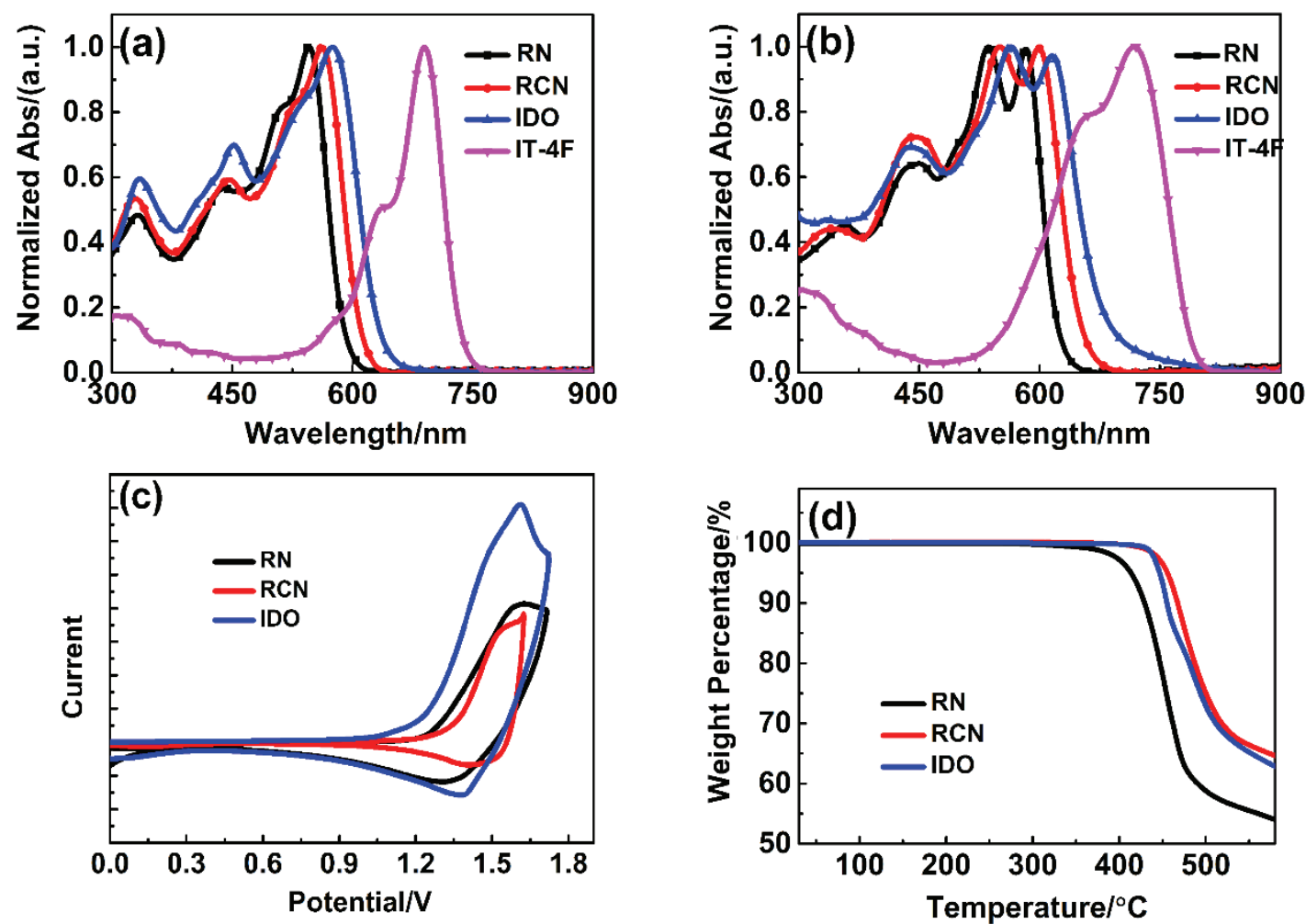

图 2 三种 TriBDT-T 小分子材料及本工作中所使用的受体材料 IT-4F 在氯仿溶液中的吸收光谱(a)与相应的 薄膜吸收光谱(b); TriBDT-T 小分子给体材料的循环伏安法测试图(c)与热重分析(TGA)测试(d) *

Fig. 2 UV-Vis absorption spectra of TriBDT-T SM materials and the acceptor IT-4F in chloroform solution(a) and the thin films (b); (c) Cyclic voltammetry curve of the TriBDT-T SM materials.

(d) TGA plots of TriBDT-T SM materials.

* (c) Cyclic voltammetry curve of the TriBDT-T films on a glassy carbon electrode in $0.1 \mathrm{~mol} \cdot \mathrm{L}^{-1} \mathrm{Bu}_{4} \mathrm{NPF}_{6}$ acetonitrile solution at a scan rate of $20 \mathrm{mV} \cdot \mathrm{s}^{-1}$ (calibrated by the $\mathrm{Fc} / \mathrm{Fc}+$ redox couple). (d) TGA plots of TriBDT-T SM materials with a heating rate of $10^{\circ} \mathrm{C} \cdot \mathrm{min}^{-1} \mathrm{under}_{2}$ atmosphere.

具有不同的影响, 一般来说拉电子能力越强, 吸收 光谱红移越明显 $22,28,38$ 。

如图 $2 b$ 所示, 三种小分子给体在固体薄膜的 吸收光谱中均出现了十分明显的吸收肩峰, 分别 位于 585、598 和 $616 \mathrm{~nm}$ 处, 这些吸收峰一般可 归因于由固态薄膜中分子间 $\pi-\pi$ 相互作用而产生 的 $\pi-\pi^{*}$ 跃迁 ${ }^{28,39,40}$ 。另外, RN、RCN 和 IDO 的溶 液消光系数相似, 分别为 $4.96 \times 10^{4} 、 4.56 \times 10^{4}$ 和 $5.22 \times 10^{4} \mathrm{~mol}^{-1} \cdot \mathrm{L} \cdot \mathrm{cm}^{-1}$ 。由此可见, 对于 A-D-A 型小分子给体材料而言, 不同 $\mathrm{A}$ 型端基的拉电子
能力可以影响其吸光性能。同时, 小分子受体 IT4F 的吸收光谱范围为 500-800 $\mathrm{nm}$, 与三种小分子 给体的吸收光谱范围基本互补, 这一点有助于在 器件中获得尽可能宽的光谱响应范围。

光伏器件的电压 $\left(V_{\mathrm{oc}}\right)$ 由给体材料的最高占有 分子轨道(HOMO) 和受体材料的最低未占分子轨 道(LUMO)的能级差决定, 因此对特定的受体材料 而言, 给体材料的 HOMO 能级越深, 相应光伏器 件的电压将会越高。在本工作中, 我们采用电化学 循环伏安法 $(\mathrm{CV})$ 来确定三种小分子给体材料的分 
子能级。在 $\mathrm{CV}$ 测量中, 使用二茂铁/二茂铁 $(\mathrm{Fc} / \mathrm{Fc}+)$ 氧化还原对作为内标。如图 $2 \mathrm{c}$ 所示, $\mathrm{RN}$ 、 $\mathrm{RCN}$ 和 IDO 的 HOMO 能级分别为 $-5.47 、-5.54$ 、 $-5.44 \mathrm{eV}$, 均具备较深的 HOMO 能级。根据文献 报道, 非富勒烯型受体材料 IT-4F 的 HOMO 能级 与 LUMO 能级为 -5.66 和 $-4.14 \mathrm{eV}^{5}$, 低于三种小 分子给体的 HOMO 和 LUMO 能级, 可以提供足 够的激子解离驱动力, 并且与三种给体材料在分 子能级方面形成良好的匹配, 因此, 从吸收光谱的 互补性和分子能级的匹配性两方面都表明 IT-4F 在本工作中作为受体材料是一个良好的选择。从 $\mathrm{CV}$ 测量结果中也可以看出, RCN 的 HOMO 能级 为三者中最低, 而 RN 与 IDO 的 HOMO 能级类 似, 因此可以预测, 当与相同的受体材料 IT- $4 \mathrm{~F}$ 共 混制备器件时, 基于 $\mathrm{RCN}$ 的光伏器件的电压应当 是三者之中最高值。

我们采用差示扫描量热法(DSC)对三种小 分子给体材料的结晶度进行了测试(见图 S1, Supporting Information), 结果表明 RN 的结晶性优 于 RCN、IDO。

\section{2 热重分析(TGA)}

我们对三个小分子给体材料进行了热重分析 测试, 结果如图 $2 \mathrm{~d}$ 所示, $\mathrm{RN} 、 \mathrm{RCN}$ 与 IDO 失重 $5 \%$ 时的热分解温度分别为 $413 、 456$ 和 $447^{\circ} \mathrm{C}$, 均 大于 $410^{\circ} \mathrm{C}$, 由此可见三者都具有较好的热稳定

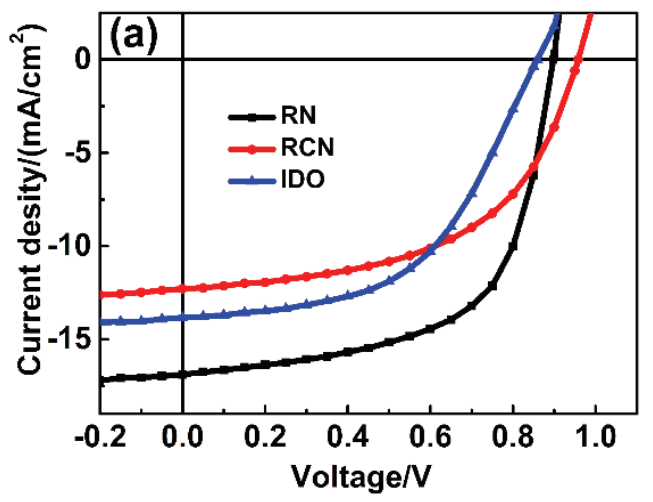

性, 在器件加工过程中不会因温度导致失活变质。

\section{3 光伏性能}

在本工作中, 我们分别采用 RN、RCN 和 IDO 为给体材料, 以 IT-4F(结构见图 S2, Supporting Information)为受体材料共混制备活性层, 其中用 氯仿作为溶剂, 使用 $\mathrm{ITO} / \mathrm{MoO}_{3}(10 \mathrm{~nm}) /$ TriBDT$\mathrm{T}$ : IT-4F/Al (100 nm) 正向结构制备光伏器件。通过 改变给受体质量比 $(\mathrm{D} / \mathrm{A})$ 和调节活性层的厚度, 获得最佳光伏性能参数。随后, 通过采用四氢呋喃 (THF) 进行溶剂退火(SVA), 进一步提高了具有最 佳 $\mathrm{D} / \mathrm{A}$ 质量比均为 $1: 1$ 和活性层厚度(约 $80 \mathrm{~nm}$ ) 的器件的光伏性能。经过上述器件优化工艺后, 基 于三种小分子给体材料的器件电流密度-电压 $(J-$ $V)$ 曲线与相应光伏性能参数分别列于图 $3 \mathrm{a}$ 和表 2 。 如表 2 所示, 经过优化器件制备工艺, 基于 RN:IT4F、 RCN:IT-4F 和 IDO:IT-4F 的器件的能量转换效 率分别为 $9.25 \% 、 6.31 \%$ 和 $6.18 \%$ 。三者的开路电 压分别为 $0.898 、 0.957$ 和 $0.859 \mathrm{~V}$, 其变化趋势与 前文中电化学测试结果推论相符; 相比而言, 基于 $\mathrm{RN}$ 的光伏器件的短路电流 $\left(J_{\mathrm{sc}}\right)$ 与填充因子 $(\mathrm{FF})$ 为 三者之中最高值, 分别为 $16.88 \mathrm{~mA} \cdot \mathrm{cm}^{-2}$ 和 $61.05 \%$, 因此得到了最高的能量转换效率; 基于 IDO:IT-4F 的光伏器件的短路电流稍高于基于 RCN:IT-4F 的光伏器件, 而前者的开路电压与填充 因子稍低于后者, 因而他们的能量转换效率相对

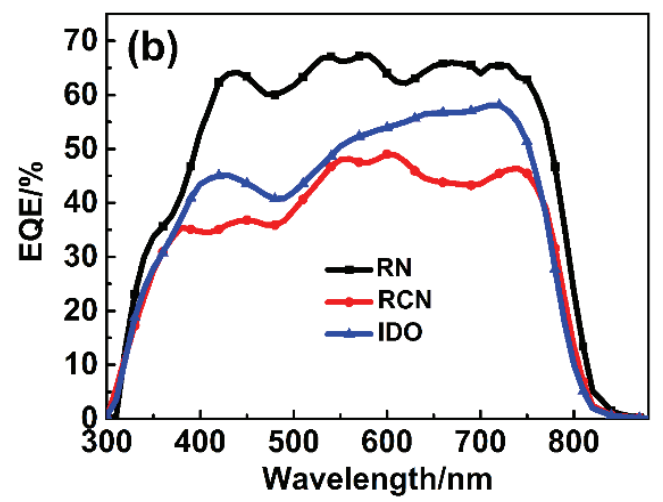

图 3 基于 SM:IT-4F 光伏器件在最优制备条件下的的 $J-V$ 曲线(a)及相应的外量子效率(EQE) (b)

Fig. 3 The $J-V$ curves of the OSC devices based on the SM:IT-4F system with the optimal fabrication conditions (a) and the corresponding EQE curves (b).

表 2 基于三种 TriBDT-T 小分子给体的光伏器件的性能参数

Table 2 Photovoltaic properties of the OSCs based on the three small molecules.

\begin{tabular}{ccccccc}
\hline Active layer & Thickness/nm & SVA $/ \mathrm{s}$ & $V_{\mathrm{oc}} / \mathrm{V}$ & $J_{\mathrm{sc}} /\left(\mathrm{mA} \cdot \mathrm{cm}^{-2}\right)$ & $\mathrm{FF}$ \\
\hline RN:IT-4F & 83 & 30 & $0.90(0.90 \pm 0.00)$ & $16.88(16.21 \pm 0.48)$ & $0.61(0.62 \pm 0.01)$ & $9.25(9.09 \pm 0.12)$ \\
RCN:IT-4F & 85 & 30 & $0.96(0.97 \pm 0.01)$ & $12.30(12.22 \pm 0.53)$ & $0.54(0.52 \pm 0.03)$ & $6.31(6.10 \pm 0.16)$ \\
IDO:IT-4F & 82 & 30 & $0.86(0.85 \pm 0.02)$ & $13.83(13.47 \pm 0.26)$ & $0.52(0.53 \pm 0.01)$ & $6.18(6.05 \pm 0.08)$ \\
\hline
\end{tabular}

a The average values were obtained over 8 devices. 
较低也较为接近。此外我们针对最佳器件的外量 子效率(EQE)进行了测试, 如图 3b 所示, 三者都 表现出较宽的光谱响应范围(300-800 nm), 然而基 于 RN:IT-4F 的器件相对于基于 IDO:IT-4F 和 RCN:IT-4F 的器件表现出更高的 EQE 响应值, 这 说明 RN:IT-4F 体系有助于光电流的生成, 因此其 短路电流为三者中最高值, 从混合膜的迁移率中 也可得出相同结论(见图 S3 与表 S1, Supporting Information)。

\section{4 活性层形貌}

针对三种器件在光伏参数方面的巨大差异, 我们使用原子力显微镜(AFM)测量基于 RN:IT$4 \mathrm{~F} 、 \mathrm{RCN}: \mathrm{IT}-4 \mathrm{~F}$ 和 IDO:IT-4F 在最佳光伏效率条件 下的活性层共混薄膜的形貌, AFM 的相应相位和 高度图像如图 4 所示, 三者的均方根表面粗粘度
(RMS) 分别为 $2.98 、 7.41$ 和 $6.91 \mathrm{~nm}$ 。一般认为, 活性层中激子扩散半径较小(约为 $10 \mathrm{~nm}$ ), 因此共 混薄膜中给受体的相区尺寸过大的话, 不利于激 子有效解离。AFM 分析表明, RN:IT-4F 共混薄膜 表现出较为理想的活性层微观形貌, 即图 $4 \mathrm{a}, \mathrm{b}$, 薄膜中存在比较明显的相分离结构, 相区尺寸较 小, 有利于激子的有效扩散与传输; 而在 RCN:IT4F (图 4d, e) 和 IDO:IT-4F (图 4g, h) 活性层薄膜 中, 我们可以观察到直径可达 $100 \mathrm{~nm}$ 的非常大的 相区尺寸, 不利于激子解离, 因此相对而言, 基于 RCN:IT-4F 和 IDO:IT-4F 的光伏器件的短路电流 与填充因子较低。随后, 我们通 过透射电子显微 镜(TEM)进一步确认了共混膜的微结构和形貌, 如 图 4 所示。TEM 结果与 AFM 测试结果基本一致, 在 RCN:IT-4F 和 IDO:IT-4F 薄膜中存在相对较强
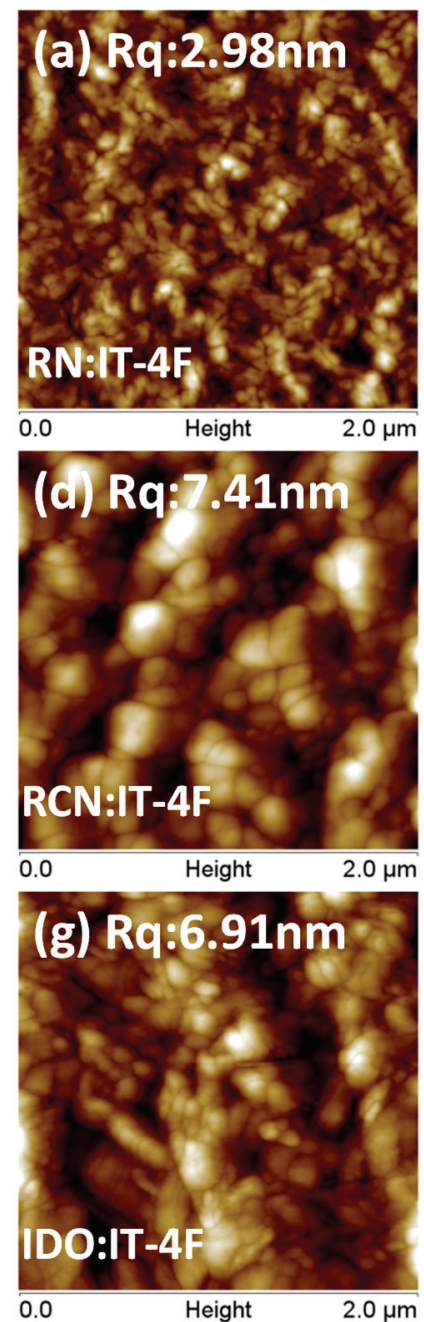
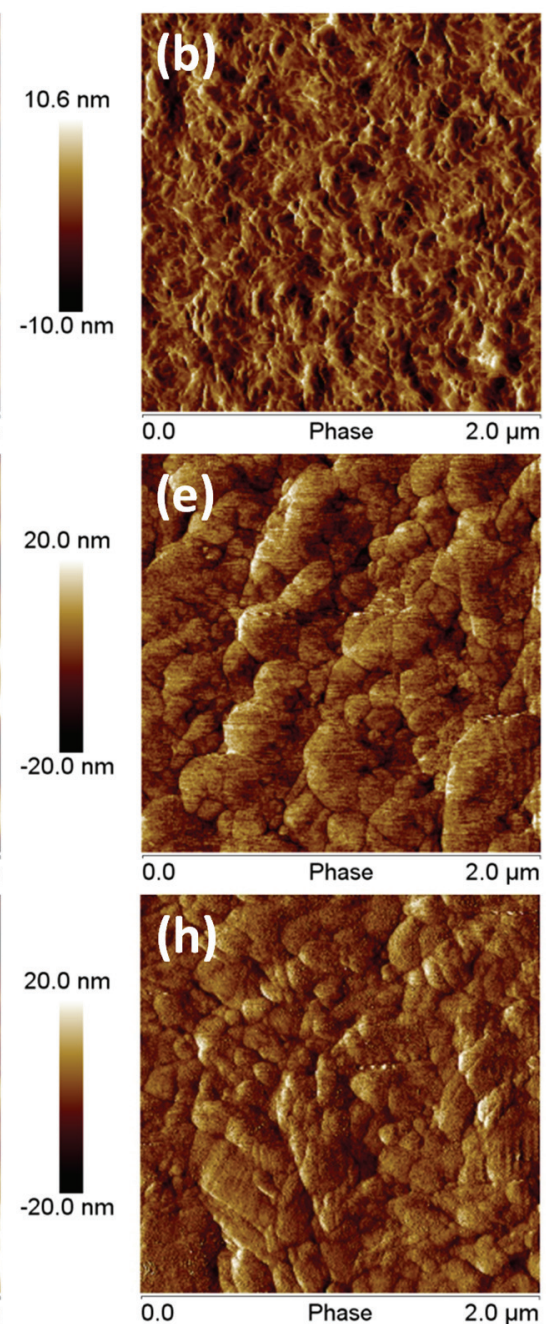
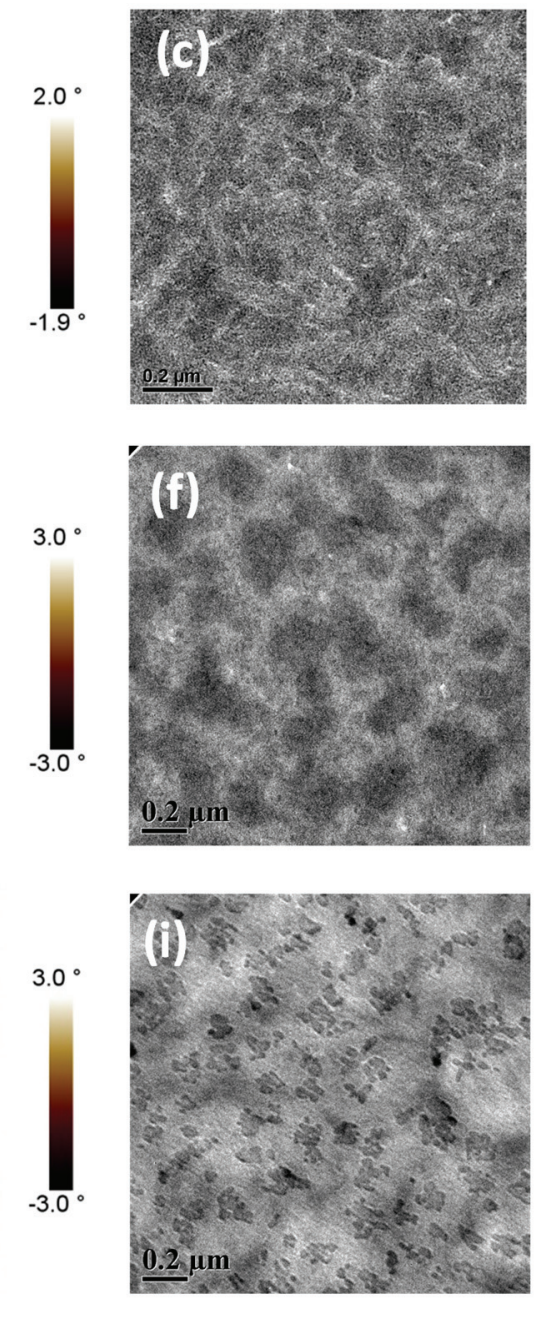

图 4 TriBDT-T:IT-4F 在最优条件下的 AFM 高度图相图和 TEM 图

Fig. 4 AFM images and TEM images of TriBDT-T:IT-4F at optimal conditions.

AFM height images of (a) RN:IT-4F and (d) RCN:IT-4F and (g) IDO:IT-4F blend films. AFM phase images of (b) RN:IT-4F and (e) RCN:IT-4F and (h) IDO:IT-4F blend films. TEM images of (c) RN:IT-4F and (f) RCN:IT-4F and (i) IDO:IT-4F blend films. 
的聚集, 意味着给受体之间的相容性较差。而 RN:IT-4F 呈现出较为规则的互穿网络结构和一定 尺寸的纳米尺寸相分离, 可以增加给体/受体界面 的电荷传输和分离效率, 从而得到更高的 $J_{\mathrm{sc}}$ 和 $\mathrm{FF}$ 以及能量转换效率。

\section{4 结论}

在本工作中, 我们以三联苯并二噻吩 (TriBDT- $\mathrm{T}$ )为 $\mathrm{D}$ 型单元、分别以罗丹宁、氧基罗丹 宁和1,3-狮二酮为 $\mathrm{A}$ 型端基, 设计并合成了具有 $\mathrm{A}-$ D-A 型结构的三种小分子给体材料 $\mathrm{RN} 、 \mathrm{RCN}$ 和 IDO。研究表明, 三种小分子的热分解温度基本一 致, 但由于 $\mathrm{A}$ 型端基的拉电子能力不同, 因此相应 给体材料的光学性能和电化学性能略有不同。随 后我们构建了由 RN、RCN、IDO与非富勒烯受体 IT-4F共混制备的非富勒烯全小分子太阳能电 池, 经过器件工艺优化, 基于 RN:IT-4F的光伏器件 的能量转换效率可以达到 $9.25 \%$, 而基于 RCN:IT$4 \mathrm{~F}$ 与IDO:IT-4F的能量转换效率仅为 $6 \%$ 左右。通过 对器件活性层微观形貌的研究发现, 带有氰基罗 丹宁与 1,3 -苑二酮为端基的小分子给体在共混薄 膜中形成了较大聚集的相区, 不利于激子的有效 传输与分离, 因此基于二者的器件的短路电流乃 至光伏性能较低。综上所述, 对于A-D-A型小分子 给体材料而言, 其末端 $\mathrm{A}$ 型基团可以对材料的吸收 光谱、分子能级以及与受体材料共混后的微观形 貌产生非常大的影响, 因此相应器件在光伏性能 方面会表现出显著差异。

Supporting Information: available free of charge via the internet at http://www.whxb.pku.edu.cn.

\section{References}

(1) Li, G.; Zhu, R.; Yang, Y. Nat. Photonics 2012, 6 (3), 153. doi: $10.1038 /$ nphoton.2012.11

(2) Brabec, C. J.; Gowrisanker, S.; Halls, J. J.; Laird, D.; Jia, S.; Williams, S. P. Adv. Mater. 2010, 22 (34), 3839. doi: 10.1002/adma.200903697

(3) Li, Y. F. Accounts Chem Res 2012, 45 (5), 723. doi: $10.1021 / \operatorname{ar} 2002446$

(4) Yu, G.; Gao, J.; Hummelen, J. C.; Wudl, F.; Heeger, A. J. Science 1995, 270 (5243), 1789. doi: 10.1126/science.270.5243.1789

(5) Zhao, W.; Li, S.; Yao, H.; Zhang, S.; Zhang, Y.; Yang, B.; Hou, J. J. Am. Chem. Soc. 2017, 139 (21), 7148. doi: 10.1021/jacs.7b02677

(6) Yao, H. F.; Hou, J. H. Acta Polym. Sin. 2016, 11, 1468. [姚惠峰, 侯 剑辉. 高分子学报, 2016, 11,1468 .] doi: 10.11777/j.issn1000-3304.2016.16216

(7) Zhang, S. Q.; Hou, J. H. Acta Phys. -Chim. Sin. 2017, 33 (12), 2327. [张少青, 侯剑辉. 物理化学学报, 2017, 33 (12), 2327.] doi: 10.3866/PKU.WHXB201706161

(8) Li, W. N.; Yao, H. F.; Zhang, H.; Li, S. S.; Hou, J. H. Chem.-Asian J. 2017, 12 (17), 2160. doi: 10.1002/asia.201700692

(9) Nielsen, C. B.; Holliday, S.; Chen, H. Y.; Cryer, S. J.; McCulloch, I. Accounts Chem Res 2015, 48 (11), 2803.

doi: 10.1021/acs.accounts.5b00199

(10) Liang, N.; Jiang, W.; Hou, J.; Wang, Z. Mater. Chem. Front. 2017, 1 (7), 1291. doi: 10.1039/c6qm00247a

(11) Sun, K.; Xiao, Z.; Lu, S.; Zajaczkowski, W.; Pisula, W.; Hanssen, E.; White, J. M.; Williamson, R. M.; Subbiah, J.; Ouyang, J.; et al. Nat. Commun. 2015, 6, 6013. doi: 10.1038/ncomms 7013

(12) Shen, S.; Jiang, P.; He, C.; Zhang, J.; Shen, P.; Zhang, Y.; Yi, Y.; Zhang, Z.; Li, Z.; Li, Y. Chem. Mater. 2013, 25 (11), 2274. doi: $10.1021 / \mathrm{cm} 400782 \mathrm{q}$

(13) Li, M.; Liu, F.; Wan, X.; Ni, W.; Kan, B.; Feng, H.; Zhang, Q.; Yang, X.; Wang, Y.; Zhang, Y.; et al. Adv. Mater. 2015, 27 (40), 6296. doi: 10.1002/adma.201502645

(14) Zhou, J.; Zuo, Y.; Wan, X.; Long, G.; Zhang, Q.; Ni, W.; Liu, Y.; Li, Z.; He, G.; Li, C.; et al. J. Am. Chem. Soc. 2013, 135 (23), 8484. doi: $10.1021 / \mathrm{ja} 403318 \mathrm{y}$

(15) Li, M.; Ni, W.; Wan, X.; Zhang, Q.; Kan, B.; Chen, Y. J. Mater. Chem. A 2015, 3 (9), 4765. doi: 10.1039/c4ta06452f

(16) Deng, D.; Zhang, Y.; Yuan, L.; He, C.; Lu, K.; Wei, Z. Adv. Energy Mater. 2014, 4 (17), 1400538. doi: 10.1002/aenm.201400538

(17) Gao, L.; Zhang, J.; He, C.; Zhang, Y.; Sun, Q. J.; Li, Y. F. Sci. ChinaChem. 2014, 57 (7), 966. doi: 10.1007/s11426-014-5114-y

(18) Badgujar, S.; Lee, G. Y.; Park, T.; Song, C. E.; Park, S.; Oh, S.; Shin, W. S.; Moon, S. J.; Lee, J. C.; Lee, S. K. Adv. Energy Mater. 2016, 6 (12), 1600228. doi: 10.1002/aenm.201600228

(19) Zhang, S.; Yang, L.; Liu, D.; He, C.; Zhang, J.; Zhang, Y.; Hou, J. Sci. China-Chem. 2017. doi: 10.1007/s11426-017-9121-0

(20) Cho, M. J.; Park, G. E.; Park, S. Y.; Kim, Y. U.; Choi, D. H. RSC Adv. 2017, 7 (62), 38773. doi: 10.1039/c7ra06879d

(21) Oliva, M. M.; Riano, A.; Arrechea-Marcos, I.; Ramos, M. M.; Gomez, R.; Algarra, M.; Ortiz, R. P.; Navarrete, J. T. L.; Segura, J. L.; Casado, J. J. Phys. Chem. C 2016, 120 (40), 23276. doi: 10.1021/acs.jpcc.6b08123

(22) Wang, W.; Shen, P.; Dong, X.; Weng, C.; Wang, G.; Bin, H.; Zhang, J.; Zhang, Z. G.; Li, Y. ACS Appl. Mater. Interfaces 2017, 9 (5), 4614. doi: 10.1021/acsami.6b14114

(23) Qiu, B.; Xue, L.; Yang, Y.; Bin, H.; Zhang, Y.; Zhang, C.; Xiao, M.; Park, K.; Morrison, W.; Zhang, Z. G.; et al. Chem. Mater. 2017, 29 , 7543. doi: 10.1021/acs.chemmater.7b02536 
(24) Yao, H.; Cui, Y.; Yu, R.; Gao, B.; Zhang, H.; Hou, J. Angew. Chem., Int. Ed. 2017, 56 (11), 3045. doi: 10.1002/anie.201610944

(25) Duan, R. M.; Cui, Y.; Zhao, Y. F.; Li, C.; Chen, L.; Hou, J. H.; Wagner, M.; Baumgarten, M.; He, C.; Mullen, K. ChemSusChem 2016, 9 (9), 973. doi: 10.1002/cssc. 201501626

(26) Zhang, J.; Zhu, X. W.; He, C.; Bin, H. J.; Xue, L. W.; Wang, W. G.; Yang, Y. K.; Yuan, N. Y.; Ding, J. N.; Wei, Z. X.; et al. J. Mater. Chem. A 2016, 4 (30), 11747. doi: 10.1039/c6ta03695c

(27) Tang, W.; Huang, D.; He, C.; Yi, Y.; Zhang, J.; Di, C.; Zhang, Z.; Li, Y. Org. Electron. 2014, 15 (6), 1155. doi: 10.1016/j.orgel.2014.03.005

(28) Zhang, Q.; Kan, B.; Liu, F.; Long, G.; Wan, X.; Chen, X.; Zuo, Y.; Ni, W.; Zhang, H.; Li, M.; et al. Nat. Photonics 2014, 9 (1), 35. doi: $10.1038 /$ nphoton.2014.269

(29) Mercier, L. G.; Mishra, A.; Ishigaki, Y.; Henne, F.; Schulz, G.; Bauerle, P. Org. Lett. 2014, 16 (10), 2642. doi: 10.1021/o1500809e

(30) Lin, Y.; Wang, J.; Zhang, Z. G.; Bai, H.; Li, Y.; Zhu, D.; Zhan, X. Adv. Mater. 2015, 27 (7), 1170. doi: 10.1002/adma.201404317

(31) Yang, Y.; Zhang, Z. G.; Bin, H.; Chen, S.; Gao, L.; Xue, L.; Yang, C.; Li, Y. J. Am. Chem. Soc. 2016, 138 (45), 15011. doi: $10.1021 /$ jacs.6b09110

(32) Yao, H.; Chen, Y.; Qin, Y.; Yu, R.; Cui, Y.; Yang, B.; Li, S.; Zhang, K.; Hou, J. Adv. Mater. 2016, 28 (37), 8283. doi: 10.1002/adma.201602642

(33) Lin, Y.; Li, T.; Zhao, F.; Han, L.; Wang, Z.; Wu, Y.; He, Q.; Wang, J.; Huo, L.; Sun, Y.; et al. Adv. Energy Mater. 2016, 6 (18), 1600854. doi: 10.1002/aenm.201600854

(34) Yao, H.; Ye, L.; Hou, J.; Jang, B.; Han, G.; Cui, Y.; Su, G. M.; Wang, C.; Gao, B.; Yu, R.; et al. Adv. Mater. 2017, 29 (21). doi: 10.1002/adma.201700254

(35) Yang, L.; Zhang, S.; He, C.; Zhang, J.; Yao, H.; Yang, Y.; Zhang, Y.; Zhao, W.; Hou, J. J. Am. Chem. Soc. 2017, 139 (5), 1958. doi: $10.1021 /$ jacs.6b11612

(36) Ni, W.; Li, M.; Wan, X.; Zuo, Y.; Kan, B.; Feng, H.; Zhang, Q.; Chen, Y. Sci. China-Chem. 2014, 58 (2), 339. doi: 10.1007/s11426-014-5220-x

(37) Kim, J.; Yun, M. H.; Anant, P.; Cho, S.; Jacob, J.; Kim, J. Y.; Yang, C. Chemistry 2011, 17 (51), 14681. doi: 10.1002/chem.201101258

(38) Cheng, Y. J.; Ho, Y. J.; Chen, C. H.; Kao, W. S.; Wu, C. E.; Hsu, S. L.; Hsu, C. S. Macromolecules 2012, 45 (6), 2690. doi: $10.1021 / \mathrm{ma} 202764 \mathrm{v}$

(39) Liu, Y.; Chen, C. C.; Hong, Z.; Gao, J.; Yang, Y. M.; Zhou, H.; Dou, L.; Li, G.; Yang, Y. Sci. Rep. 2013, 3, 3356. doi: 10.1038/srep03356

(40) Yong, W.; Zhang, M.; Xin, X.; Li, Z.; Wu, Y.; Guo, X.; Yang, Z.; Hou, J. J. Mater. Chem. A 2013, 1 (45), 14214. doi: $10.1039 / \mathrm{c} 3 \operatorname{ta} 12229 \mathrm{~h}$ 